\title{
AS EMISSÕES OTOACÚSTICAS NO DIAGNÓSTICO DIFERENCIAL DAS PERDAS AUDITIVAS INDUZIDAS POR RUÍDO
}

\section{Otoacoustic Emissions in the differential diagnosis of the noise-induced auditory losses}

\author{
Miriam de Souza Basílio Coelho ${ }^{(1)}$, Joice Regina da Silva Ferraz ${ }^{(2)}$, \\ Elizabeth de Oliveira Crepaldi Almeida ${ }^{(3)}$, Nelson de Almeida Filho ${ }^{(4)}$
}

\begin{abstract}
RESUMO
Tema: artigos relacionados a emissões otoacústicas no diagnóstico diferencial da perda auditiva induzida por ruído (PAIR). Objetivos: evidenciar a importância da utilização das emissões otoacústicas no diagnóstico diferencial da perda auditiva induzida por ruído (PAIR). Conclusão: confirmou-se que tanto as emissões otoacústicas transientes (EOAT) quanto às emissões otoacústicas por produto de distorção (EOAPD) são úteis no diagnóstico precoce da PAIR sendo que as EOAT se revelam mais sensíveis às mudanças temporárias de limiar que as EOAPD e as EOAPD se destacam mais eficazes no diagnóstico precoce da PAIR em indivíduos com audição aparentemente normal por captar respostas nas frequências mais agudas que são primeiramente atingidas na PAIR. Acredita-se, ainda, que este estudo possa beneficiar a população quanto à prevenção e controle da PAIR e incentivar a criação de políticas públicas que contribuirão com a prevenção das alterações auditivas dentro do âmbito ocupacional.
\end{abstract}

DESCRITORES: Diagnóstico Precoce; Perda Auditiva; Ruído Ocupacional

\section{INTRODUÇÃO}

A audição é fundamental à vida, e o órgão responsável por ela é o ouvido atualmente denominado orelha, de acordo com a Nomenclatura Anatômica Brasileira ${ }^{1}$.

A orelha é divida em três partes: orelha externa, orelha média e orelha interna. Dessas partes a mais importante é a orelha interna principalmente a

(1) Aluna do curso de Graduação em Fonoaudiologia da Pontifícia Universidade Católica de Campinas, PUCCAMP, Campinas, SP.

(2) Aluna do curso de Graduação em Fonoaudiologia da Pontifícia Universidade Católica de Campinas, PUCCAMP, Campinas, SP.

(3) Fonoaudióloga; Docente Titular do curso de Fonoaudiologia da Pontifícia Universidade Católica de Campinas, PUCCAMP, Campinas, SP; Pesquisadora colaboradora do Grupo de Estudo dos Aspectos Moleculares da Perda Auditiva da Universidade Estadual de Campinas, UNICAMP, Campinas, SP; Doutora em Educação.

(4) Estudante de Medicina.

Conflito de interesses: inexistente sua porção anterior, a cóclea, que é rica em líquidos de sódio e potássio e aloja o Órgão de Corti, que é um órgão essencial para recepção da audição, formado basicamente por células de sustentação e células secundárias ciliadas externas e internas (estereocílios), ambas localizadas e fixadas sobre a membrana basilar em toda sua extensão e unidas por ligamentos de elastina. As células externas mais longas se fixam superiormente em uma membrana denominada membrana tectória, enquanto as mais curtas não fazem esse contato ${ }^{\text {? }}$.

Em estado de excitação os estereocílios se alongam e se inclinam determinando assim, a abertura dos canais para as trocas de íons - cascata de eventos. Essas entradas são como links que se forem rompidos interrompem o tráfego sanguíneo e levam à apoptose. Quando ocorre uma perda temporária da audição essas entradas se mantêm abertas e por isso ocorre a recuperação auditiva. Já na exposição prolongada ao ruído acima de 85 $\mathrm{dB}$, as células perdem a motilidade e se soltam da 
membrana basilar levando a uma exaustão metabólica, ou seja, ocorre uma exitotoxidade (intoxicação da célula por ruído), com consequente apoptose ${ }^{2}$.

Devido as suas propriedades biomecânicas, as células externas têm capacidade de dois tipos de contração: rápida e lenta. Essa capacidade de contração constitui um amplificador coclear e tem grande importância no mecanismo de amplificação do estímulo sonoro para determinar o funcionamento das células ciliadas internas, que são as unidades receptoras e codificadoras cocleares e têm papel importante na seletividade frequencial da cóclea ${ }^{3}$.

A contração rápida é a base do mecanismo ativo induzido pelos deslocamentos dos estereocílios, sendo, esta, o suporte das emissões otoacústicas (EOA). A contração lenta controla a tonicidade das células ciliadas externas (CCE) e regula as propriedades mecânicas da membrana basilar. O sistema eferente medial e a CCE provocam o mecanismo das contrações lentas e estes atenuam a contração rápida, ou seja, o amplificador coclear funciona como um amortecedor do amplificador, reduzindo desta forma, as EOA. Dessa forma, as emissões otoacústicas se originam da atividade das células ciliadas externas e resultam do processo biomecânico dessas células ${ }^{2,3}$.

Kemp ${ }^{4}$ foi quem observou inicialmente as emissões otoacústicas, definindo-as como uma liberação de energia sonora procedente da cóclea, a qual percorre a orelha média e chega ao meato acústico externo. Com isso, evidenciou-se que essas emissões encontram-se presentes em todos os indivíduos que apresentam normalidade funcional da orelha; já em indivíduos nos quais foram encontradas perdas auditivas acima de $20-30 \mathrm{~dB}$ as emissões otoacústicas não são detectadas ${ }^{5}$.

As EOA podem ser de dois tipos: espontâneas e evocadas. As espontâneas são sons naturais emitidos pela cóclea na ausência de estímulo acústico e estão presentes em $50 \%$ dos indivíduos com audição normal ${ }^{4}$. As evocadas são respostas cocleares geradas de acordo com o estímulo apresentado e que podem ser classificadas como: transientes (EOAT), estímulo-frequência e produto de distorção (EOAPD) ${ }^{6}$. Estão presentes em indivíduos que apresentam um funcionamento normal da cóclea e sua presença significa que a orelha média e a cóclea estão respondendo normalmente ao estímulo.

É um teste que prevê um método objetivo, fácil, rápido e não-invasivo ${ }^{7,8}$, é utilizado para monitorar a função da cóclea ${ }^{9} \mathrm{e}$ apresenta maior sensibilidade para detectar lesão das células ciliadas externas ${ }^{10}$. As EOA asseguram um diferencial no diagnóstico por se tratar de um teste prático que avalia a função coclear de forma segura e são sensíveis aos estágios iniciais de alterações na função coclear e, para qualquer diagnóstico precoce, é importante saber que as emissões são registradas com limiar normal ou perda auditiva inferior a $30 \mathrm{~dB}$. A intensidade das EOA cresce com a intensidade da estimulação até 20 ou $30 \mathrm{~dB}^{7}$ - importante em perda auditiva induzida por ruído (PAIR).

Entende-se por PAIR uma lesão coclear irreversível, provocada por prolongada exposição ao ruído de grande intensidade ( $85 \mathrm{~dB}$ ), que pode vir acompanhada de zumbido e é caracterizada por uma curva audiológica do tipo entalhe, atingindo, geralmente, as frequências de 3,4 ou $6 \mathrm{KHz}{ }^{11} \mathrm{e}$, com o agravamento da lesão, estende-se às demais frequências, as quais levam mais tempo para serem atingidas ${ }^{12}$.

Nos últimos anos tem se observada grande preocupação quanto ao diagnóstico precoce das perdas auditivas e, entre elas, a PAIR, que foi um tema polêmico no campo ocupacional ${ }^{13}$. Essa preocupação pode estar relacionada com o fato de a PAIR ser uma doença silenciosa e que se instala ao longo do tempo. Entretanto, existe uma suscetibilidade individual para tal manifestação, que pode ser de origem genética ou por tempo e nível de exposição ao ruído, além de maior predisposição para a orelha esquerda ${ }^{2}$.

Estudos têm evidenciado que o ruído associado a exposição a agentes químicos podem causar e/ ou intensificar a possibilidade de aparecimento de perdas auditivas ${ }^{14}$.

É indispensável aos profissionais da saúde auditiva ocupacional, a utilização de um método simples e eficaz para esse diagnóstico ${ }^{15}$. A justificativa para o uso de um teste diferencial é o fato do ruído muito intenso causar lesões nas estruturas do Órgão de Corti, degenerando inicialmente as células ciliadas externas em maior quantidade e, posteriormente, as células ciliadas internas. Como o exame das EOA capta o funcionamento da cóclea pelas respostas das células externas, o teste pode revelar a integridade ou a alteração dessas estruturas, antes das mesmas apontarem irregularidade no exame de audiometria tonal, garantindo ainda, uma avaliação segura nos casos de perdas auditivas funcionais.

As EOA têm sido utilizadas para o diagnóstico de diversas doenças principalmente as EOAPD ${ }^{16}$, já que as EOAT são utilizadas, normalmente, para triagem auditiva neonatal (TAN). Já foi exposto que EOA, também, podem ser aplicadas em programas de conservação auditiva com a proposta de detecção precoce do prejuízo da audição causado por exposições ocupacionais ${ }^{17}$.

Estudos indicam a importância das EOAPD e EOAT no diagnóstico precoce de PAIR e confirmam 
essa tese em trabalhos que mostram a probabilidade da exposição ao ruído ocupacional provocar alterações nos registros das EOAPD ${ }^{8,15,18}$. Outros autores também afirmam a necessidade da utilização da EOAPD, na qual é analisada a atividade da cóclea em frequências específicas ${ }^{19,20}$.

Os indivíduos expostos a ruído intenso devem ser acompanhados auditivamente. Esse acompanhamento poder ser feito por meio da audiometria tonal e pelas EOAT ou EOAPD ${ }^{19}$. As EOAPD são importantes, pois interrompem o ciclo de cascata de eventos (abertura e fechamento dos canais) ${ }^{2}$. O uso das EOAT no diagnóstico precoce de PAIR pode ser muito eficaz, já que detecta mudanças temporárias do limiar (TTS) frente a níveis elevados de pressão sonora ${ }^{6,21}$.

Em estudos realizados com músicos, as EOA revelaram mais alterações que exames de audiometria tonal ${ }^{22,23}$. A avaliação auditiva realizada apenas pela audiometria tonal pode não retratar a real situação do funcionamento da cóclea dos indivíduos expostos ao ruído ${ }^{6}$. O exame deve ser realizado apenas como rastreio e os profissionais devem lançar mão de outras provas diagnósticas, pois muitas patologias podem apresentar perdas auditivas na faixa dos $4 \mathrm{kHz}$, dessa forma, seria imperícia do profissional dar um diagnóstico baseado apenas na audiometria tonal ${ }^{24}$.

Para a avaliação auditiva ocupacional, normalmente, os profissionais são guiados pelas políticas públicas nacionais em vigor. Essas legislações até o momento não estabelecem a utilização das EOA como procedimento de prevenção, conservação e detecção das PAIR, como é o caso da portaria 19, de 09 de abril de 1998, da NR 7 do Ministério do Trabalho e Emprego ${ }^{11}$, no entanto, o teste das EOA pode ser aplicado como exame complementar, já que se trata de uma avaliação simples, rápida e de importante aplicação.

A prevenção da PAIR é a melhor opção para a saúde auditiva no âmbito ocupacional e, uma vez instalada, cabe ao especialista detectá-la precocemente evitando dessa forma sua evolução.

Com a finalidade de evidenciar a vantagem da utilização das emissões otoacústicas no diagnóstico diferencial das perdas auditivas induzidas por ruído, este trabalho propôs uma reflexão dos artigos relativos ao tema escolhido, entre o período de 01/2005 a 12/2007, em revistas científicas na área de otorrinolaringologia e fonoaudiologia, baseadas no critério CAPES - QUALIS ${ }^{25}$.

\section{MÉTODOS}

Foram escolhidos os artigos relacionados com a área audiológica, referentes ao tema proposto, publicados no período de 01/2005 a 12/2007, em todas as revistas nacionais específicas das áreas de Otorrinolaringologia e Fonoaudiologia, baseando-se nos critérios do CAPES - QUALIS - Classificação de Periódicos, Anais, Revistas e Jornais, seguindo sua nova estratificação, com base em 2007 e vigor a partir de 03/2009. Essa nova estratificação compreende uma avaliação realizada por diversas áreas de conhecimento (Educação Física, Medicina, Odontologia, Letras, Engenharia...); dessa forma, expôs-se uma avaliação geral e detaIhada dessas áreas (Tabela 2).

Dos periódicos selecionados, três faziam parte da área de otorrinolaringologia $(A, B$ e $C)$ e quatro da área de fonoaudiologia (D, E, F e G) e estão descritos a seguir de acordo com sua classificação atual no CAPES - QUALIS.

\section{Revistas de Otorrinolaringologia}

De todos os periódicos nacionais existentes na área de Otorrinolaringologia, nenhum apresenta nota $A 1$ e A2 no WEBQUALIS.

- Periódico A

Categoria Atual: CAPES QUALIS - Estratos variando de $\mathrm{B} 1$ a $\mathrm{C}$ de acordo com a área de avaliação.

- Periódico B

Categoria Atual: CAPES QUALIS - Estratos variando de B3 a B5 de acordo com a área de avaliação.

- Periódico C

Categoria Atual: CAPES QUALIS - Estrato B5 de acordo com área de avaliação.

\section{Revistas de Fonoaudiologia}

De todos os periódicos nacionais existentes na área de Fonoaudiologia, nenhum apresenta nota A1 e A2 no WEBQUALIS.

- Periódico D

Categoria Atual: CAPES QUALIS - Estratos variando de $\mathrm{B} 1$ a $\mathrm{B} 5$ de acordo com a área de avaliação.

- Periódico E

Categoria Atual: CAPES QUALIS (base 2007 com vigor a partir de 03/2009). Estratos variando de B1 a B5 de acordo com a área de avaliação.

- Periódico F

Categoria Atual: CAPES QUALIS (base 2007 com vigor a partir de 03/2009). Estratos variando de B3 a B5 de acordo com a área de avaliação.

- Periódico G

Categoria Atual: CAPES QUALIS (base 2007 com vigor a partir de 03/2009). Estratos variando de B1 a B5 de acordo com a área de avaliação. 
Realizou-se uma análise descritiva e qualitativa das revistas descritas com o intuito de avaliar a opinião dos diferentes autores sobre a utilização das EOAT e EOAPD no diagnóstico precoce da PAIR.

\section{REVISÃO DA LITERATURA}

Os resultados encontrados durante a busca e a seleção dos artigos totalizaram cinco, os quais se destacam a seguir:

- Periódico A - total de dois artigos.

1) MARQUES. F. P; COSTA, E. A. Exposição ao ruído ocupacional: alterações no exame de emissões otoacústicas (2006).

2) BARROS, S. M. S; FROTA, S; ATHERINO, C. C. T; OSTERNE, F. A eficiência das emissões otoacústicas transientes e audiometria tonal na detecção de mudanças temporárias nos limiares auditivos após exposição a níveis elevados de pressão sonora (2007).
- Periódico $\mathbf{C}$ - total de um artigo.

1) MARTINS, C. H. F; VASSOLER, T.M.F; BERGONSE G. F. R; ALVARENGA, K. F. COSTA, O. A. Emissões otoacústicas e potencial evocado auditivo de tronco encefálico em trabalhadores expostos a ruído e ao chumbo (2007).

- Periódico F - total de dois artigos.

1) FIORINI, A. C. PARRADO-MORAN. M. E. S. Emissões otoacústicas - produto de distorção: estudo de diferentes relações de níveis sonoros no teste em indivíduos com e sem perdas auditivas (2005).

2) MAIA, J. R. F. Estudo da audição de músicos de rock and roll (2007).

Os periódicos encontrados foram classificados, ainda, de acordo com o tipo de teste utilizado para o procedimento diagnóstico durante a avaliação (Tabela 1).

Os demais periódicos (B, D, E e G) não foram selecionados por não apresentarem artigos relacionados ao tema principal.

Tabela 1 - Classificação dos artigos conforme o tipo de teste diagnóstico

\begin{tabular}{|c|c|c|c|c|c|c|c|}
\hline Periódico / Autor & EOAT & EOAPD & $\begin{array}{c}\text { Áudio } \\
\text { T. }\end{array}$ & Imitanc. & PEATE & $\begin{array}{l}\text { Diag. } \\
\text { Precoce }\end{array}$ & $\begin{array}{l}\text { Diag. } \\
\text { Tardio }\end{array}$ \\
\hline $\begin{array}{l}\text { Periódico A } \\
\text { (MARQUES; COSTA, } \\
\text { 2006) }\end{array}$ & & $\square$ & $\square$ & & & $\square$ & \\
\hline $\begin{array}{l}\text { Periódico A } \\
\text { (BARROS et al.,2007) }\end{array}$ & $\square$ & & $\square$ & $\square$ & & $\square$ & \\
\hline $\begin{array}{l}\text { Periódico C } \\
\text { (MARTINS et al., 2007) }\end{array}$ & $\square$ & & & & $\square$ & & $\square$ \\
\hline $\begin{array}{l}\text { Periódico F } \\
\text { (FIORINI; PARRADO- } \\
\text { MORAN, 2005) }\end{array}$ & & $\square$ & & $\square$ & & $\square$ & $\square$ \\
\hline $\begin{array}{l}\text { Periódico F } \\
\text { (MAIA, 2007) }\end{array}$ & $\square$ & $\square$ & $\square$ & $\square$ & & $\square$ & \\
\hline \multicolumn{8}{|c|}{$\begin{array}{l}\text { EOAT - Emissões otoacústicas Transientes } \\
\text { EOAPD - Emissões otoacústicas produto de distorção } \\
\text { Áudio T. - Audiometria tonal } \\
\text { Imitanc. - Imitanciometria } \\
\text { PEATE - Potenciais evocados auditivo de tronco encefálico } \\
\text { Diag. Precoce - Diagnóstico Precoce } \\
\text { Diag. Tardio - Diagnóstico Tardio }\end{array}$} \\
\hline
\end{tabular}

Nas avaliações audiológicas da PAIR, até pouco tempo atrás eram utilizados somente testes de audiometria tonal, no entanto, já é tema de discussão de grandes estudos nessa área que, a audiometria tonal, em muitos casos, pode não revelar uma perda auditiva em seu início pelo fato da PAIR atingir inicialmente a base da cóclea, comprometendo dessa forma, as altas frequências. Desde as descobertas de Kemp ${ }^{4}$ sobre as EOA, têm se estudado sobre as respostas das células ciliadas externas diante de estimulação sonora e muitas pesquisas foram realizadas nesse sentido. 
Tabela 2 - Estratificação de Periódicos Conforme a Área de Avaliação

\begin{tabular}{|c|c|c|}
\hline Periódico & Estratificação & Área de Avaliação \\
\hline \multirow{16}{*}{ A } & B1 & Educação Física \\
\hline & B1 & Interdisciplinar \\
\hline & B2 & Engenharias II \\
\hline & B3 & Engenharias III \\
\hline & B3 & Letras / Lingüística \\
\hline & B3 & Medicina I \\
\hline & B3 & Medicina II \\
\hline & B3 & Odontologia \\
\hline & B3 & Saúde Coletiva \\
\hline & B4 & Ciências Biológicas I \\
\hline & B4 & Engenharias I \\
\hline & B4 & Farmácia \\
\hline & B5 & Ciências Biológicas III \\
\hline & $\mathrm{C}$ & Biotecnologia \\
\hline & C & Ensino de Ciências e Matemática \\
\hline & $\mathrm{C}$ & Química \\
\hline $\mathrm{B}$ & B5 & Educação Física \\
\hline \multirow{8}{*}{ C } & B3 & Educação \\
\hline & B3 & Educação Física \\
\hline & B3 & Interdisciplinar \\
\hline & B3 & Saúde Coletiva \\
\hline & B5 & Engenharias I \\
\hline & B5 & Engenharias III \\
\hline & B5 & Medicina I \\
\hline & B5 & Medicina II \\
\hline \multirow{4}{*}{$\mathrm{D}$} & B3 & Interdisciplinar \\
\hline & B5 & Educação Física \\
\hline & B5 & Medicina II \\
\hline & B5 & Odontologia \\
\hline \multirow{10}{*}{$\mathrm{E}$} & B1 & Educação Física \\
\hline & B3 & Educação \\
\hline & B2 & Interdisciplinar \\
\hline & B3 & Letras / Linguística \\
\hline & B5 & Medicina I \\
\hline & B3 & Odontologia \\
\hline & B4 & Engenharias I \\
\hline & B4 & Saúde Coletiva \\
\hline & B5 & Engenharias IV \\
\hline & B5 & Medicina II \\
\hline \multirow{7}{*}{$\mathrm{F}$} & B5 & Engenharias III \\
\hline & B1 & Educação Física \\
\hline & B3 & Interdisciplinar \\
\hline & B5 & Medicina I \\
\hline & B5 & Letras / Lingüística \\
\hline & B5 & Medicina II \\
\hline & B5 & Odontologia \\
\hline \multirow{10}{*}{$\mathrm{G}$} & B5 & Engenharias III \\
\hline & B3 & Medicina II \\
\hline & B3 & Odontologia \\
\hline & B4 & Ciências Biológicas I \\
\hline & B1 & Educação Física \\
\hline & B2 & Saúde Coletiva \\
\hline & B3 & Medicina I \\
\hline & B3 & Educação \\
\hline & B1 & Letras / Linguística \\
\hline & B5 & Engenharias I \\
\hline
\end{tabular}


Nos artigos descritos a seguir, observam-se as vantagens e desvantagens dos diversos testes utilizados na esfera ocupacional.

Marques e Costa ${ }^{15}$ compararam trabalhadores de um mesmo estabelecimento que permaneciam, ou não, expostos ao ruído ocupacional, utilizando a audiometria tonal para a seleção dos indivíduos com limiares dentro da normalidade e o exame das EOAPD. Os resultados mostraram maior prevalência de ausências de respostas no teste de EOAPD em indivíduos expostos ao ruído.

Os resultados concordam com os de Garcia et al. ${ }^{21}$ em seu trabalho de comparação entre os tipos de EOA, no qual, as EOAPD mostraram ocorrência de piora significativa para as freqüências agudas. Com isso, conclui-se a importância da utilização desse exame para o diagnostico da PAIR, pois esta, afeta inicialmente as freqüências mais altas.

A pesquisa realizada por Barros et.al. ${ }^{6}$ se resume na utilização da audiometria tonal associada às EOAT na pré e pós-exposição a níveis elevados de pressão sonora, para verificação da eficiência dos testes na detecção de pequenas mudanças temporárias no limiar. A imitanciometria foi utilizada para descartar anormalidades em orelha média. $\mathrm{Na}$ comparação entre audiometria tonal e EOAT, verificou-se que ambas revelaram sensibilidade na detecção de mudanças temporárias nos limiares de audibilidade e reprodutibilidade após a exposição a níveis elevados de pressão sonora, sendo que a audiometria tonal foi mais eficiente para as frequências de 3 a $8 \mathrm{kHz}$ e as EOAT para as frequências de 1 a $4 \mathrm{kHz}$. Os resultados das EOA concordam com o estudo de Garcia et al. ${ }^{21}$ que, na análise das EOAT, revelou redução na amplitude de respostas em indivíduos avaliados após exposição à níveis elevados de pressão sonora.

A desvantagem das EOAT nesse aspecto é que, a mesma, não alcança a resposta de frequencias acima de $4 \mathrm{kHz}$.

Normalmente, os indivíduos raramente expostos a níveis elevados de pressão sonora, podem ter a recuperação auditiva após um tempo de repouso auditivo, porém, quando a exposição se torna frequente, gradualmente se instala a mudança permanente do limiar que é a PAIR.

Martins et.al. ${ }^{13}$ expõem os resultados de seu estudo sobre o nível de comprometimento da função auditiva em trabalhadores expostos ao chumbo e ao ruído e compara esses resultados com o de trabaIhadores expostos apenas ao ruído empregando na testagem auditiva o potencial evocado auditivo do tronco encefálico (PEATE) e as emissões otoacústicas transientes (EOAT). A pesquisa revelou que o chumbo não comprometeu significativamente a cóclea, porém, o PEATE se apresentou alterado significativamente nos indivíduos expostos aos dois componentes.

Nesse caso, o PEATE atua como um auxiliar na detecção de perdas neurossensoriais de trabaIhadores atuantes em locais, onde o ruído está acompanhado de substancias neurotóxicas como o chumbo, pois essas substancias, segundo 0 estudo, atingem as vias auditivas do tronco encefálico e não necessariamente as células ciliadas; por esse fato, as perdas não foram diagnosticadas no teste de EOA. Dessa forma pode-se afirmar que o tipo de perda causada pelo chumbo é retrococlear com lesão das vias auditivas centrais e o teste mais indicado para o diagnóstico exato é o PEATE que avalia a condução do som pelas vias auditivas desde a parte inicial do VIII par de nervos cranianos no seu ramo coclear, até o tronco encefálico. Isso não quer dizer que um indivíduo exposto ao chumbo e ao ruído concomitantemente, não apresente lesões em cóclea, mas, nessa pesquisa, o chumbo teve um efeito de lesão mais rápido que o ruído, contudo, esses indivíduos podem apresentar futuramente lesão em nível coclear, caso não seja controlado o nível de ruído à que estão expostos.

Para Jacob, Alvarenga e Morata ${ }^{14}$ são escassos os estudos do efeito do chumbo no sistema auditivo, além dos poucos existentes apresentarem resultados contraditórios. Em sua revisão bibliográfica no estudo sobre efeitos auditivos do chumbo em trabalhadores expostos e esse componente tóxico, não há estudos que relatem as consequências de associação do chumbo e do ruído em trabalhadores expostos a esses dois elementos, porém, a pesquisa realizada pôde concluir que os efeitos do chumbo vão além da cóclea atingindo as vias auditivas centrais. As autoras sugerem que os profissionais da saúde ocupacional considerem exposições a agentes químicos ao estudar perdas auditivas relacionadas ao trabalho.

Fiorini e Parrado-Moran ${ }^{20}$ compararam a audição de indivíduos sem perda auditiva e não expostos a ruído ocupacional com indivíduos com perda auditiva neurosensorial expostos a ruído ocupacional, por meio da audiometria tonal, imitanciometria e EOAPD e os resultados demonstraram que os indivíduos, mesmo sem queixas auditivas e sem exposição ao ruído ocupacional, obtiveram limiares tonais piores nas frequências de 6,4 e 8 $\mathrm{kHz}$ e falha na resposta de algumas frequências no teste de EOAPD. Na imitanciometria, a curva timpanométrica serviu para verificar a existência de doenças em orelha média que pudessem interferir no teste de EOA e o teste do reflexo acústico foi importante para registrar a contração do músculo estapédio, na presença do som intenso. 
O mais interessante nessa pesquisa é que os indivíduos sem queixas auditivas que apresentaram limiares tonais prejudicados e ausência de respostas nas EOAPD sem estarem expostos ao ruído ocupacional relataram vivenciar momentos de lazer ouvindo músicas em alto volume. O estudo afirmou, portanto, que o exame de EOAPD pode proporcionar dados importantes sobre agravos na cóclea, ajudando, dessa forma, o especialista a apresentar o diagnóstico.

Maia ${ }^{22}$ refere na audiometria tonal a alta prevalência do entalhe, mesmo com limiares dentro do limite da normalidade, como indicativo para o desencadeamento futuro da PAIR, denominada, em seu estudo com músicos de rock and roll, de PAINEPS (perda auditiva induzida por níveis elevados de pressão sonora).

Muitas vezes um resultado com a respectiva descrição pode passar despercebido numa avaliação audiológica em que a anamnese é pouco investigada ou até mesmo por profissionais pouco habilitados na área ocupacional.

Quanto à imitanciometria, as curvas timpanométricas apresentaram integridade de orelha média e houve ausência de reflexo na frequência de $4 \mathrm{kHz}$ em $34,8 \%$ dos participantes. A autora relaciona esse resultado ao fato da ausência do reflexo, nessa frequência específica, não apresentar relevância diagnóstica mesmo em indivíduos com orelhas normais.

Já as EOAT revelaram que $61 \%$ dos participantes apresentaram ausência de respostas na frequência de $4 \mathrm{kHz}$ e as EOAPD revelaram menor resposta de amplitude na frequência de $6 \mathrm{kHz}$, além da grande incidência de respostas ausentes nas frequências mais baixas $(0,75 \mathrm{e} 1 \mathrm{kHz})$ fato este, justificado pela autora como resultado da característica geral do rock que pode alterar as frequências mais baixas por apresentar faixa de frequência de som comprimida, amplificação de banda estreita e reverberação amplificada e reamplificada.

Verifica-se no estudo que, além das EOAPD apresentarem a frequência de $6 \mathrm{kHz}$ alterada, ocorrência que a EOAT não consegue desempenhar por captar frequências de até $4 \mathrm{kHz}$, também apresentaram ausência de respostas nas frequências mais graves pelas características próprias do estilo musical. Se a PAIR atinge inicialmente as frequências mais agudas, como já é sabido, mais uma vez aqui se confirma a eficácia das EOAPD no seu diagnóstico precoce. Sobre os riscos auditivos oferecidos por esse estilo musical, também para as frequências mais graves, pode ser necessária uma orientação diferencial e adequada a esses trabalhadores, com conscientização sobre os riscos auditivos pela exposição excessiva à música com forte intensidade.
Considerando os dados das pesquisas analisadas, observa-se que a alteração auditiva pode não ser percebida em seu início e, para esses casos, as EOA são úteis em diagnosticar precocemente os danos auditivos em estágios iniciais, com realização de um controle periódico adequado no campo ocupacional.

Os artigos, apenas cinco, aqui apresentados, discutem profundamente sobre a utilização das EOA no diagnóstico diferencial da PAIR, que têm mostrado excelente resultado diferencial nesses casos.

As EOAT são mais sensíveis às mudanças temporárias do limiar de audibilidade que as EOAPD e, as EOAPD, mais eficazes para detectar freqüências agudas ${ }^{21}$.

Quanto ao melhor tipo de EOA para o diagnóstico, deve-se levar em consideração que as EOAT captam as frequências até $4 \mathrm{Khz}$ e as EOAPD captam até $8 \mathrm{kHz}$ e por este motivo são mais sensíveis para avaliações ocupacionais pelo fato da PAIR atingir, inicialmente, as frequências mais altas.

Nem todos os profissionais vinculados à saúde auditiva ocupacional utilizam-se de métodos mais eficazes como os aqui discutidos e, em especial, de EOAPD, orientando-se apenas pelas políticas públicas em vigor, que citam a audiometria tonal como único exame para o controle da PAIR no âmbito ocupacional, como é o caso da NR7 do Ministério do Trabalho (Portaria no 19/98) 11, na qual é obrigatória a realização de audiometria em todos os funcionários expostos a ruídos ocupacionais. Todavia, até o momento não existem políticas públicas que determinem a utilização das EOA como auxiliar ou substituto da audiometria tonal nos exames periódicos dos trabalhadores. Diante disso, faz-se necessário a criação de leis que estabeleçam a utilização de testes, considerados diferenciais, no acompanhamento auditivo de profissionais expostos ao ruído ocupacional, com o objetivo de se diagnosticar precocemente a instalação irreversível da PAIR.

\section{CONCLUSÃO}

Diante dos resultados encontrados pode-se concluir que, tanto as EOAT quanto as EOAPD são úteis no diagnóstico da PAIR, sendo que as EOAT se revelam mais sensíveis às mudanças temporárias de limiar que as EOAPD e as EOAPD se destacam mais eficazes no diagnóstico precoce da PAIR em indivíduos com audição aparentemente normal, pelo fato de captar as respostas nas freqüências mais altas, que são as primeiras 
a serem atingidas pela PAIR, enquanto que a audiometria tonal revela a PAIR quando esta já se instalou permanentemente.

Acredita-se, ainda, que este estudo possa beneficiar a população quanto à prevenção e controle da PAIR e incentivar a criação de políticas públicas que contribuirão com a prevenção das alterações auditivas dentro do âmbito ocupacional.

\begin{abstract}
Background: articles related of otoacoustic emissions in differential diagnosis of noise-induced hearing loss (NIHL). Purposes: to demonstrate the importance of using otoacoustic emissions in the differential diagnosis of noise-induced hearing loss (NIHL). Conclusion: it was confirmed that both the transient-evoked otoacoustic emissions (TEOAE) and distortion-product otoacoustic emissions (DPOAE) are useful in the early diagnosis of and the TEOAE and are more sensitive to temporary alterations in the DPOAE threshold, and DPOAE stand out more effectively in the early diagnosis of $\mathrm{NIHL}$ in individuals with apparently normal hearing responses by capturing responses more acute frequencies in that are first affected in NIHL. It is also believed that this study can benefit the population on the NIHL prevention and control and foment the creation of public policies that shall contribute to the prevention of hearing impairment within the occupational field.
\end{abstract}

KEYWORDS: Early Diagnosis; Hearing Loss; Noise, Occupational

\section{REFERÊNCIAS}

1. Sociedade Brasileira de Anatomia. Nomenclatura Anatômica. Arq Cir Clin Exp. 1961; 24(3/4):1-11.

2. Meinke D, Radar TS. Incidental noise exposures in youth. $24^{\circ}$ Encontro Internacional de Audiologia, 2009. Bauru: Academia Brasileira de Audiologia; 2009.

3. Oliveira JAA. O mecanismo eletrobiomecânico ativo da cóclea. Rev Bras Otorrinolaringol. 1993 out./dez; 59(4):236-48.

4. Kemp DT. Stimulated acoustic emission from whit in the human auditory system. J Acoust Soc Am. 1978; 64(5):1386-92.

5. Kemp DT, Bray P, Alexander L, Brown AM. Acoustic emission cochleography: pratical aspects. Scand Audiol. 1986; 25(Suppl):71-95.

6. Barros SMS, Frota S, Atherino CCT, Osterne F. A eficiência das emissões otoacústicas transientes e audiometria tonal na detecção de mudanças temporárias nos limiares auditivos após exposição a níveis elevados de pressão sonora. Rev Bras Otorrinolaringol. 2007 set./out; 73(5):592-8.

7. Lopes Filho O, Campos CAH. Tratado de otorrinolaringologia. São Paulo: Roca; 1994.

8. Frota S, lório MCM. Emissões otoacústicas por produto de distorção e audiometria tonal liminar: estudo da mudança temporária do limiar. Rev Bras Otorrinolaringol. $2002 \mathrm{jan} / \mathrm{fev}$; 68(1):15-20.

9. Aquino AMCM, Massaro CAM, Tiradentes JB, Garzon JCV, Oliveira JAA. Emissões otoacústicas no diagnóstico precoce de lesão coclear na doença de Meniére. Rev Bras Otorrinolaringol. 1993 out/ dez; 68(5):761-6.

10. Silveira JAM, Brandão ALA, Rossi J, Ferreira LLA, Name MAM, Estefan P, et al. Avaliação da alteração auditiva provocada pelo uso do walkman, por meio da audiometria tonal e das emissões otoacústicas (produto de distorção) estudo de 40 orelhas. Rev Bras Otorrinolaringol. 2001 set/out; 67(5):650-4.

11. Brasil. Ministério do Trabalho. Secretaria de Segurança e Saúde no Trabalho. Portaria n.19 de 9 de abril de 1998. D. O. U. de 22/04/98 - Seção I p. 64 a 66. Acesso em: 26 mar 2009. Disponível em: http://www.mte.gov.br/legislacao/ portarias/1998/p_ 19980409_19.pdf

12. Comitê Nacional de Ruído Conservação Auditiva. Boletim $\mathrm{n}^{\circ}$ 1. Arq Int Otorrinolaringol. [periódico na internet]. 2000; 4(2). [acesso em: 07 abr 2009] Disponível em: URL: http://www.scielo. br/scielo.php? script=sci_arttext\&pid=S003472 992001000500008\&tlng=en\&lng=en\&nrm=iso

13. Martins CHF, Vassoler TMF, Bergonse GFR, Alvarenga KF, Costa OA. Emissões otoacústicas e potencial evocado auditivo de tronco encefálico em trabalhadores expostos a ruído e ao chumbo. Acta ORL. 2007 out-dez; 25(4):255-325.

14. Jacob LCB, Alvarenga KF, Morata TC. Os efeitos da exposição ocupacional ao chumbo sobre o sistema auditivo: uma revisão de literatura. Rev Bras Otorrinolaringol. 2002 jul/ago; 68(4):564-9. 
15. Marques FP, Costa EA. Exposição ao ruído ocupacional: alterações no exame de emissões otoacústicas. Rev Bras Otorrinolaringol. 2006 mai/ jun; 72(3):362-6.

16. Aringa ARD, Aringa AHBD, Juares AJC, Melo C, Filho RMP. Emissões otoacústicas por produto de distorção em crianças de 2 a 7 anos. Rev Bras Otorrinolaringol. 2004 mai/jun; 70(3):380-4.

17. Gattaz G, Wazen SRG. O registro das emissões acústicas evocadas - produto de distorção em pacientes com perda auditiva induzida pelo ruído. Rev Bras Otorrinolaringol. 2001 mar/abr; 67(2):213-8.

18. Alvarenga $\mathrm{KF}$, Jacob LCB, Martins $\mathrm{CHF}$, Costa AO, Coube CZV, Marques JM. Emissões Otoacústicas - produto de distorção em indivíduos expostos ao chumbo e ao ruído. Rev Bras Otorrinolaringol. 2003 set/out; 69(5):681-9.

19. Rocha EB, Azevedo MF, Ximenes Filho J. A. Estudo da audição de crianças de gestantes expostas ao ruído ocupacional: avaliação por emissões otoacústicas - produto de distorção. Rev Bras Otorrinolaringol. 2007 mai/jun; 73(3):359-69.

20. Fiorini AC, Parrado-Moran MES. Emissões otoacústicas - produto de distorção: estudo de diferentes relações de níveis sonoros no teste em indivíduos com e sem perdas auditivas. Rev Dist Comun. 2005 dez; 17(3):385-96.

21. Garcia AP, Snege YC, Azevedo MF, Soares E. Emissões otoacústicas evocadas transientes e emissões otoacústicas evocadas por produto de distorção em adultos normais: estudos dos desvios transitórios do limiar de audibilidade após exposição ao ruído. Pró-Fono. 1999; 11(2):53-60.

22. Maia JRF. Estudo da audição de músicos de rock and roll. Rev Dist Comun. 2007; 19(3):275.

23. Namuur FABM, Fukuda Y, Onishi ET, Toledo RN. Avaliação auditiva em músicos da orquestra sinfônica municipal de São Paulo. Rev Bras Otorrinolaringol. 1999 set/out; 65(5):390-5.

24. Costa EA, Morata TC; Kitamura S. Patologia do ouvido relacionada com o trabalho. In: Mendes R. Patologia do trabalho. 2. ed. São Paulo: Atheneu; 1999. p.1253-79

25. Capes - Qualis - Classificação de periódicos, anais, revistas e jornais. [homepage na internet. São Paulo; 2009. [acesso em: 08 mar 2009]. Disponível em: http://qualis.capes.gov.br/webqualis/ ConsultaPeriodicos.faces

DOI: 10.1590/S1516-18462010005000108

RECEBIDO EM: 20/10/09

ACEITO EM: 15/01/2010

Endereço para correspondência:

Elizabeth de Oliveira Crepaldi Almeida

Av. Maria Martins Otobone, 210

Jacareí - SP

CEP: 12306-700

E-mail: nelson_almeida@uol.com.br 